\title{
Estimation of cumulative exposure to organophosphate sheep dips in a study of chronic neurological health effects among United Kingdom sheep dippers
}

\author{
D Buchanan, A Pilkington, C Sewell, S N Tannahill, M W Kidd, B Cherrie, J F Hurley
}

\begin{abstract}
Objectives-To derive a method for retrospectively estimating cumulative exposure to organophosphate (OP) pesticides among a cross section of United Kingdom sheep dippers, as part of a wider epidemiological study of neurological abnormality within this group of workers.

Methods-A hygiene study of dipping sessions at 20 farms using diazinon based dips was carried out by two experienced occupational hygienists. Observations on the exposure of people to concentrate and dilute dip were recorded throughout each dipping session, together with the other relevant factors including the use and condition of protective clothing. Concentrations of urinary metabolites of diazinon were used to measure actual exposure to OPs. To estimate exposure in the subsequent epidemiological study, an occupational exposure history questionnaire was developed using results from the hygiene
\end{abstract} study and an empirical exposure model.

Results-In the hygiene study, increased urinary metabolites were associated with the handling of concentrate dip and exposure to dilute dip wash through splashing. Very few dippers wore the recommended protective clothing. The handling of concentrate dip was the principal source of exposure to OPs. Dipping task was used as a surrogate for splashing of dilute dip in retrospective exposure estimation. In the epidemiological study, cumulative exposure to OP sheep dips was highly correlated with the total number of dipping days, but not with age.

Conclusions-Sheep dip concentrate is the most important source of OP exposure among sheep dippers and estimates of exposure to OPs during routine dipping should take due account of exposure to concentrate dip as well as to the dilute dip wash. The observed use of recommended protective clothing by most subjects was insufficient to allow a proper empirical assessment of its effectiveness. (Occup Environ Med 2001;58:694-701)

Keywords: organophosphate pesticides; sheep dip; exposure model

This paper describes the results of the first phase of a study of United Kingdom sheep dippers commissioned in 1995 jointly by the United Kingdom Health and Safety Executive (HSE), Department of Health (DoH), and the Ministry of Agriculture, Fisheries and Food (MAFF). The overall aim of the study was to investigate whether there was a relation between chronic low level exposure to organophosphates (OPs) in sheep dip formulations and neurophysiological health effects. ${ }^{1}$ The objective of the first phase of the study was to derive a method for retrospectively estimating cumulative low level exposure to OPs among a cross section of United Kingdom sheep dippers.

In the United Kingdom, OPs have been present in sheep dips since the 1960s when organochlorines were phased out due to concerns about public health. They are used to give effective control over the spread of sheep scab, ticks, blowfly, and lice. Usage increased during the 1970s after sheep scab emerged as a problem in England and Wales in 1972, and compulsory national dipping was introduced throughout the United Kingdom in 1976. Compulsory dipping was relaxed in 1992.

Estimation of long term exposure to pesticides in epidemiological studies has proved difficult. ${ }^{2}$ Less attention has been paid to methods of measuring dermal exposure, the main route of exposure in these studies, compared with methods for inhalation exposure. ${ }^{3}$ Use of pesticides is generally irregular and time within a job is not an accurate predictor of actual exposure. ${ }^{2}$ Quantitative indices of exposure have not often been used and where they have, they have typically been based on job exposure matrices (JEMs) with matrix weights based on expert knowledge of the level of exposure within tasks. ${ }^{4}$ In a recent study into the effects of chronic exposure among United Kingdom sheep dippers, ${ }^{5}$ the estimated total number of sheep dipped (flock size $\times$ dips per year $\times$ years) was used as a surrogate for cumulative exposure.

Biological monitoring, in particular the inhibition of plasma cholinesterase, has been used extensively to measure acute and recent exposure to Ops. ${ }^{6}$ This method was used to assess the relation between recent exposure and acute symptoms in a group of Welsh sheep farmers. ${ }^{7}$ However, these methods cannot be used to measure long term retrospective exposure to Ops. ${ }^{6}$

Niven $e t a l^{8}$ reported a hygiene assessment among sheep dippers which noted that, although concentrations of urinary metabolites 
of OPs were generally low, large variations in working methods and exposure to dip wash were found, even among dippers on the same farm. Results suggested that the main source of exposure among dippers was due to the handling of concentrate dip, with exposure due to splashing of dilute dip from the dipping bath less significant.

This paper describes firstly a hygiene study of sheep dippers designed to allow an empirical model of exposure to OPs to be derived for use in the main epidemiological phase of the study. Secondly the development and application of a retrospective exposure questionnaire based on the empirical model are described.

\section{Methods}

HYGIENE STUDY

Twenty farms, covering a broad range of size and type of sheep farm, were recruited with help from the National Farmers Union, Scottish Agricultural College, and dip suppliers. Only farms that used diazinon sheep dips were considered because this was historically one of the most common OP active ingredients and a validated method of analysis of urinary metabolites existed for this compound. Each farm was visited on a day when dipping was taking place by two experienced occupational hygienists.

A proforma was devised, modified from that used by Niven et $a l,{ }^{8}$ to allow the hygienists to record general information while attending each dipping session. General information on dipping practices was recorded for each farm, including flock size, OP products, and dipping facilities. Individual workers involved in the dipping session were numbered and wore coloured armbands for ease of recognition. Tasks carried out by individual workers throughout the day were noted. At farms with their own dipping facilities, three well defined dipping tasks have previously been described: the plunger (or paddler), chucker, and the helper. ${ }^{8}$ Helpers, who are normally the most distant from the dip bath, round up the sheep ready for the chucker to usher them one by one into the dip bath. The plunger stands beside the bath and plunges each sheep under the surface with a plunging implement, or sometimes with feet and hands.

In an extension to Niven $\mathrm{et} \mathrm{al},{ }^{8}$ the proforma was structured to allow a greater degree of measurement of the principal routes of exposure previously identified: concentrate handling, splashing with dilute dip from the dip bath, and indirectly through ingestion (while smoking, drinking, or eating). Observations were recorded for each person throughout the day.

Concentrate, which typically contains diazinon as an active ingredient at $16 \%$ or $60 \%$ by weight, is added to water in the bath initially in amounts necessary to ensure correct working strength (about $0.40 \mathrm{ml} / 1$ ), with regular replenishment. Each event in which concentrate was handled was recorded for each person, together with information on the protective gloves worn at the time of handling and whether skin was washed after handling. The degree of protection afforded by the protective gloves at the time of handling (none, poor, fair, good) was also noted.

At four evenly spaced periods throughout the dipping session, visual assessments of the degree of splashing on 10 body regions (hands, face, hair, front and back torso, lower and upper arms, lower and upper legs, and feet) were made, noting whether areas were dry, splashed, or soaked. The level of protection (none, poor, fair, good) offered by a person's own and protective clothing on each area of the body was also noted. A simple time weighted splash score, representing the degree of exposure to dilute dip wash, was calculated from the product of time exposed (duration of dipping) and average exposure intensity for each person as follows:

time weighted splash score $=t x \frac{\sum_{i} \sum_{j} s_{i j}}{n_{i}}$

where $t$ is the time spent dipping by a person, $s_{i j}$ represents the degree of splashing on body region $j$ at time $i$ and $n_{i}$ is the number of times splashing was observed during the session (up to a maximum of four). The degree of splashing on each body region, $s_{i j}$, was based on a visual inspection of the workers and was assigned values: $0=$ none, $1=$ splashed, $2=$ soaked. A time weighted splash score corrected for protective clothing worn at observation was derived by setting $s_{i j}$ to zero on regions of the body where protective clothing was worn and judged to be effective.

Direct ingestion of droplets is possible during dipping but difficult to measure. The potential for indirect ingestion of OPs was measured by recording each occasion when a person took food or drink, or smoked while dipping, including whether gloves were removed and hands washed.

Three samples of urine were collected from each individual dipper: one before the start of the dipping session, one immediately after dipping, and a further sample early the following morning. Before the study, people were sent sufficient Sterilin containers, polythene bags, and labels, plus an information sheet on collection and storage of samples. Samples were collected in person from farms on the day after the observed dipping session and frozen until analysis. Urinary metabolites diethylphosphate (DEP) and diethylthiophosphate (DETP) were analysed with high resolution gas chromatography with flame photometric detection. ${ }^{9}$ Urinary concentrations were corrected for creatinine, measured with the Jaffe reaction, and expressed as $\mathrm{nmol} / \mathrm{mmol}$ of creatinine. Samples that were overdiluted (creatinine $<0.5 \mathrm{~g} / \mathrm{l}$ ) or overconcentrated (creatinine $>3 \mathrm{~g} / \mathrm{l}$ ) were rejected..$^{10}$ An estimate of exposure to OP during the observed dipping session was assessed from the increment in the concentration of urinary metabolites (DEP+DEPT) from the sample before dipping to the sample the next morning. A few subjects with very high concentrations before dipping (who had been dipping in the days before the study) were excluded before 


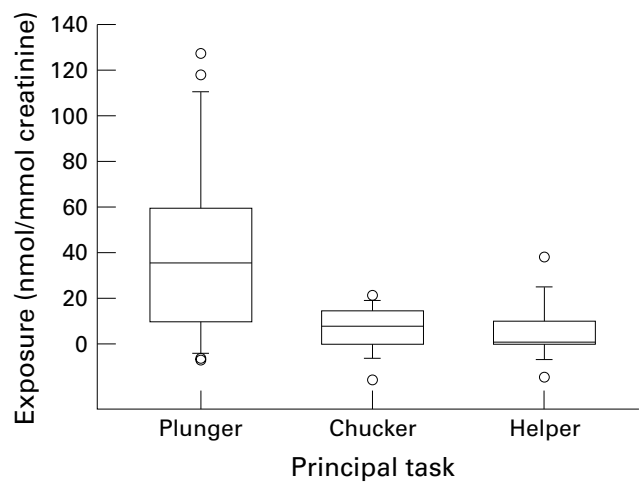

Figure 1 Box plots of measured exposure (increment of concentrations of $D E P+D E P T$ in urinary samples from before dipping to the next morning) by principal sheep dipping task.

analysis to ensure an unbiased estimate of exposure due solely to the dipping session under observation.

Standard linear regression and analysis of variance (ANOVA) methods were used to analyse the relation between dipping factors and measured exposure with the Genstat statistical software package. ${ }^{11}$ Scatter plots and linear correlation coefficients were used to assess the degree of association among the various cumulative exposure indices. The robustness of parameter estimates was investigated by repeating analyses omitting potential outliers and influential values.

\section{Results}

HYGIENE STUDY

Twenty farms were recruited and 60 subjects were surveyed during dipping, each on a single day. Valid urinary samples were provided before and after dipping by 50 subjects. Four subjects with high urinary concentrations

Table 1 Mean (SEM) exposure variables by principal dipping task

\begin{tabular}{lrrr}
\hline & \multicolumn{2}{l}{ Principal task } & \\
\cline { 2 - 4 } Variable & Plunger & Chucker & Helper \\
\hline Subjects (n) & \multicolumn{1}{c}{17} & 13 \\
Urinary metabolites & $40.8(9.8)$ & $7.3(3.0)$ & $5.3(3.7)$ \\
Events handling concentrate† & $7.1(1.6)$ & $0.7(0.1)$ & $0.2(0.4)$ \\
Duration dipped (h) & $4.9(0.4)$ & $3.8(0.4)$ & $5.1(0.5)$ \\
Time weighted splash score $\neq$ & $55.9(5.3)$ & $27.7(4.9)$ & $9.2(3.4)$ \\
Eating or drinking events & $2.8(0.5)$ & $1.7(0.4)$ & $2.8(0.4)$ \\
Smoking events & $0.9(0.4)$ & $0.2(0.5)$ & $0.8(0.2)$ \\
\hline
\end{tabular}

^Increment in urinary concentration of DEP+DEPT from before dipping to the next morning (nmol/mmol creatinine).

†Uncorrected for protective gloves.

$\ddagger$ Uncorrected for protective clothing. before dipping ( $>40 \mathrm{nmol} / \mathrm{mmol}$ ) were excluded. A further four people, who had not been observed for the entire dipping session, were also omitted.

Figure 1 shows the distribution of actual exposures across the three main principal tasks as measured by concentrations of urinary metabolites. Plungers clearly experienced the highest exposure, whereas chuckers had only marginally higher exposure than helpers on average.

Table 1 shows the summary statistics for various exposure variables by principal task. Plungers had the highest observed exposure to contact with the concentrate, as measured by the number of events during which concentrate was handled. The plungers observed in this study generally had sole responsibility for handling concentrate throughout the dipping session. Due to the nature of the task, plungers also had highest observed exposure to dilute dip wash, as measured by the time weighted splash score. Helpers, who were furthest from the bath, tended to have a lower observed exposure to dilute dip wash than chuckers who could be exposed to a high level of splashing as sheep entered the dip bath.

Table 2 shows the estimated linear regression coefficients for variables representing the principal routes of exposure, with and without correcting exposure for protective clothing worn at observation. From all data, variables representing concentrate handling and dip wash splashing were highly significant. Results with and without correction for protective clothing were similar as very few subjects were observed to wear the recommended protective clothing. For example, in $93 \%$ of the 132 observed events handling concentrate, the subject did not wear the recommended protective gloves (unlined PVC or nitrile heavy duty gauntlets). Also, the correct and uncorrected splash scores were highly correlated $(r=0.85 ; \mathrm{p}<0.001)$.

The number of ingestion events, in isolation, was not a significant predictor of exposure, with or without including smoking events. This was also the case if an effect was only expected among those already with high exposure of the hands. An interaction term for events in which concentrate was handled and ingestion events (after fitting both as main effects) was not significant, whether smoking events were included $(\mathrm{p}=0.92)$ or not $(\mathrm{p}=0.61)$.

Figure 2 shows a scatter plot of measured exposure against number of events in which

Table 2 Estimated values of variables from linear regression of increment in urinary metabolites (DEP+DETP) against each exposure variable individually using the full data set and two subsets, one excluding a potentially influential value (subject 10/2), one excluding three potential outliers (subjects 15/1, 17/2, and 23/1)

\begin{tabular}{|c|c|c|c|c|c|c|}
\hline \multirow[b]{2}{*}{ Variable } & \multicolumn{2}{|c|}{ All subjects $(n=42)$} & \multicolumn{2}{|c|}{ Excluding subject $10 / 2(n=41)$} & \multicolumn{2}{|c|}{$\begin{array}{l}\text { Excluding subjects } 15 / 1,17 / 2 \text {, and } \\
23 / 1(n=39)\end{array}$} \\
\hline & Variable & p Value & Variable & $p$ Value & Variable & p Value \\
\hline Handling concentrate & $4.40(0.61)$ & $<0.001$ & $4.04(0.80)$ & $<0.001$ & $4.04(0.50)$ & $<0.001$ \\
\hline Corrected for handling concentrate* & $4.76(0.71)$ & $<0.001$ & $4.26(0.92)$ & $<0.001$ & $4.37(0.59)$ & $<0.001$ \\
\hline Time weighted splash score & $0.81(0.14)$ & $<0.001$ & $0.66(0.14)$ & $<0.001$ & $0.70(0.14)$ & $<0.001$ \\
\hline Corrected for time weighted splash score & $1.54(0.35)$ & $<0.001$ & $1.07(0.42)$ & 0.014 & $1.30(0.32)$ & $<0.001$ \\
\hline Ingestion events & $3.07(2.99)$ & 0.31 & $3.55(2.54)$ & 0.17 & $-0.30(2.85)$ & 0.92 \\
\hline Ingestion and smoking events & $-0.78(2.16)$ & 0.72 & $-0.23(1.86)$ & 0.90 & $-2.09(1.97)$ & 0.30 \\
\hline
\end{tabular}

^Excludes events while wearing "good" protective gloves.

†Excludes splashing while wearing "good" protective clothing. 


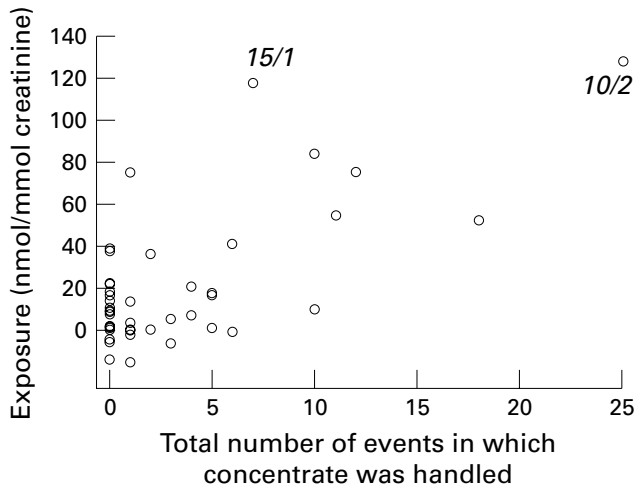

Figure 2 Scatter plot showing the relation between measured exposure (increment of concentrations of $D E P+D E P T$ from urinary samples before dipping to the next morning) and number of events in which concentrate was handled. Two subjects have been identified, one as a possible outlier (15/1) and one a potentially influential value (10/2).

concentrate was handled. Concentrate handling, without adjustment for gloves, explained the highest proportion of variation between person in urinary metabolites $\left(R^{2}=56 \%\right)$. Omitting the most influential data point from the regression analysis (subject 10/2) produced a similar linear coefficient that was also highly significant (table 2). One potential outlier (subject 15/1) had been suspected of handling concentrate without being directly observed; therefore exposure to concentrate may have been underestimated.

After adjusting for handling concentrate, exposure was, on average, greater among those who used dips containing $60 \%$ diazinon rather than $16 \%$ diazinon, although the mean adjusted difference of $9.6 \mathrm{nmol} / \mathrm{mmol}$ creatinine (SE 7.7) was not significant. It was not possible to investigate empirically the effect of protective gloves as only a single person in the analysis data set wore the recommended protective gloves while handling concentrate. However, adjusting for events in which concentrate was handled, there was no evidence that subjects who wore gloves of any quality (poor, fair, or good) for greater than $50 \%$ of events in which concentrate was handled had lower exposure than those who did not.

Results suggested a simple linear model for exposure $(E X P)$ during a single dipping session:

$$
E X P=\alpha+\beta C+\gamma S
$$

where $C$ and $S$ represent number of events in which concentrate was handled and time weighted splash score for dilute dip respectively, and $\alpha, \beta$, and $\gamma$ are parameters.

The results of fitted regression models for urinary concentrations of metabolites that

Table 3 Multiple linear regression of increments in urinary metabolites (DEP + DETP) against the number of events handling concentrate and the uncorrected time weighted splash score using the full data set and a subset excluding three potential outliers (subjects 17/2, $23 / 1$, and 15/1) ( $\alpha, \beta$, and $\gamma$ refer to model parameters in equation (2))

\begin{tabular}{|c|c|c|c|c|}
\hline Subjects included & $\begin{array}{l}\text { Subjects } \\
(n)\end{array}$ & $\begin{array}{l}a(\text { intercept }) \\
\text { mean }(S E M)\end{array}$ & $\begin{array}{l}\beta \text { (concentrate) } \\
\text { mean }(S E M)\end{array}$ & $\begin{array}{l}\gamma(\text { splash }) \\
\text { mean }(S E M)\end{array}$ \\
\hline All subjects & 42 & $-2.4(5.1)$ & $3.1(0.8)$ & $0.4(0.2)$ \\
\hline $\begin{array}{l}\text { Excluding subjects } 17 / 2 \text {, } \\
23 / 1 \text {, and } 15 / 1\end{array}$ & 39 & $3.0(4.6)$ & $3.6(0.7)$ & $0.2(0.2)$ \\
\hline
\end{tabular}

included both linear terms for concentrate handling $(C)$ and dip wash splash $(S)$ are shown in table 3. After adjusting for handling concentrate, dilute dip splash remained significant $(p=0.02)$. This was plausible as splash was simply a dilute form of the concentrate dip.

Table 3 also shows the regression parameters after omitting the three outlying data points (subjects 15/1, 17/2, 23/1). This parameter set was expected to be more robust to outlying observations and hence more representative of most dippers in the study. The intercept parameter $a$, corresponding to zero overall exposure, was estimated to be close to zero in both models, and was subsequently omitted.

Using the robust parameter set, one concentrate handling event was predicted to be equivalent to a time weighted splash score of 18 . The median time weighted splash score among the study group was 33, equal to about two events in which concentrate was handled, and therefore a much lower effect on exposure than the median of eight handling events among concentrate handlers. Therefore, among those dippers principally responsible for handling concentrate throughout a session, concentrate handling was the most important source of exposure.

RETROSPECTIVE ESTIMATION OF EXPOSURE IN EPIDEMIOLOGICAL STUDY

This section describes how the results of this study of sheep dip methods were used to estimate retrospectively cumulative exposure to OPs among a cross section of working sheep dippers in the wider epidemiological study. ${ }^{1}$

\section{Use of surrogates for exposure}

To allow the exposure model in equation (2) to be used to estimate exposures retrospectively by questionnaire, it was necessary to investigate other factors which were related to the model input variables and which could be accurately assessed with a questionnaire. Using these factors, surrogate values could then be substituted for the concentrate $(C)$ and splash $(S)$ components in the model to allow estimation of exposure for any given past dipping scenario.

A single dipper was generally responsible for handling concentrate throughout a session. This dipper was most often found to be the plunger, although this was not necessarily a rule that could be applied more widely. An earlier study had reported that chuckers also often acted as principal concentrate handlers. ${ }^{8}$

Sheep dip manufacturers commonly specified that baths be regularly topped up with concentrate after every 50 or 60 sheep had been dipped so flock size was considered as a method of estimating exposure to concentrate. A positive association was found between the total number of events in which concentrate was handled and the total number of sheep dipped at each session, equal to one event for evry 100 sheep dipped. An alternative method considered was to substitute the mean number of handling events per session (8.0) among the principal concentrate handlers.

Factors associated with exposure to dilute dip splash were examined with ANOVA of 
Table 4 Predicted mean (95\% CI) time weighted splash scores from two factor ANOVA model that included principal task and bath type (fitted on the log scale)

\begin{tabular}{|c|c|c|c|}
\hline \multirow[b]{2}{*}{ Principal task } & \multicolumn{3}{|l|}{ Bath type } \\
\hline & Linear & Circular & Mobile \\
\hline Plunger & $66(52$ to 81$)$ & 49 (35 to 66$)$ & 39 (26 to 55$)$ \\
\hline Chucker & 44 (32 to 57$)$ & $31(19$ to 45$)$ & 23 (12 to 36$)$ \\
\hline Helper & $10(5$ to 17$)$ & $4(1$ to 11$)$ & $2(0$ to 6$)$ \\
\hline
\end{tabular}

uncorrected time weighted splash scores (log transformed). Principal task was found to explain the largest proportion of the variation in splashing with dilute dip wash. Bath type (linear, circular, or mobile) was also found to have a significant effect. Mean splash scores by task and bath type, predicted with a two factor ANOVA model, additive on the log scale, are shown in table 4 . This confirmed that plungers and chuckers were exposed to splashing to a greater degree than helpers and suggested that dippers who used circular baths (five farms) and mobile baths (five farms) were exposed to slightly less splashing than dippers using the more common linear, or straight swim, bath (10 farms).

The effect on the exposure model prediction of substituting surrogate values for splash and concentrate, based on the above factors, is shown in table 5 . This shows the linear correlation between the observed and predicted increment in urinary metabolites using the original splash and concentrate variables in equation (2) and two pairs of surrogate input variables. The extended surrogate inputs were, for principal concentrate handlers, the number of sheep dipped divided by 100, and, for splash exposure, the task by bath means in table 4 . The simple surrogate inputs were, for principal concentrate handlers, a mean of eight events, and for splash exposure, the task specific means for linear baths in table 4 . Predictions using both the robust and non-robust model parameter sets from table 3 were compared, but as they did not differ discernibly, the robust parameter set was preferred for model prediction. Both sets of surrogate variables gave predictions that were positively correlated with observations The simple surrogate model $(r=0.56)$ performed marginally better than the extended surrogate model $(r=0.52)$ although both sets of predictions were, as expected, less correlated with observed exposure than the original exposure variables $(r=0.78)$.

Independent validation of the exposure model was possible with similar data for a sample of 51 dippers pooled from two earlier hygiene studies. ${ }^{812}$ Linear correlation with observed increment in urinary metabolites was poor when the full validation data set was used. However, omitting one subject with a very high urinary increment $(226.5 \mathrm{nmol} / \mathrm{mmol}$ creatinine) and previously identified as an outlier, ${ }^{12}$ resulted in a significant correlation between observed and predicted exposure using the simple surrogate variables $(r=0.39 ; \mathrm{p}=0.005)$. This positive correlation between observed and predicted exposure, which can be seen in figure 3, was slightly higher than the correlation using

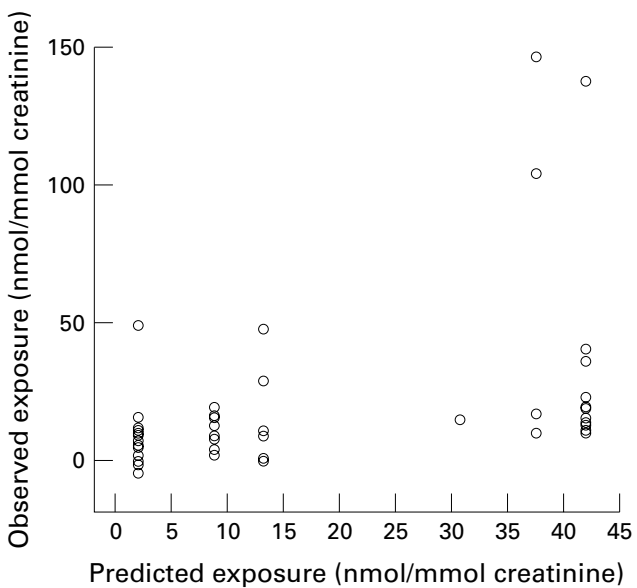

Figure 3 Relation between observed and predicted exposure (increment of concentrations of DEP+DEPT from urinary samples before dipping to the next morning) among an independent sample of subjects from two earlier studies $^{812}$. Predicted exposure uses surrogates for concentrate handling (the mean among principal handlers) and dilute dip splash (task based mean splash scores).

the extended surrogate variables $(r=0.33$; $\mathrm{p}=0.021$ ).

\section{Exposure history questionnaire}

The hygiene study results were used to inform the construction of an exposure history questionnaire for use in the later exposure-response study of a cross section of sheep dippers.

The first part of the questionnaire comprised a full job history with dates, job title, and employer, starting with the current job and back to 1970, before which OPs were not commonly used in sheep dip formulations. For each job that involved sheep dipping, a second part was completed that included more detail on dipping with OP sheep dips. For each job, an estimate of the number of dipping days using OP dips was requested. This was most easily derived by the interviewee providing, for each year in the job, the number of dips per year and the number of dipping days per flock. Subjects were asked on what proportion of dipping days they acted as plunger, chucker, and helper. Additional questions about the use of waterproof clothing (trousers and footwear) and use of plunging implements were included. Subjects were asked on what proportion of dipping days they were principally responsible for handling concentrate, including how often they

Table 5 Linear correlation ( $r$ ) between observed and predicted exposure comparing predictions using the original concentrate and splash exposure variables, and two sets of surrogate input variables for splash and concentrate (parameters $\beta$ and $\gamma$ correspond to regression estimates shown in table 3)

\begin{tabular}{|c|c|c|}
\hline \multirow[b]{2}{*}{$\begin{array}{l}\text { Exposure variables } \\
\text { used in prediction }\end{array}$} & \multicolumn{2}{|c|}{ Model parameter sets } \\
\hline & $\begin{array}{l}\beta=3.6, \gamma=0.2 \\
\text { (robust) }\end{array}$ & $\begin{array}{l}\beta=3.1, \gamma=0.4 \\
\text { (non-robust) }\end{array}$ \\
\hline Original variables* & 0.78 & 0.79 \\
\hline Extended surrogates $\dagger$ & 0.52 & 0.52 \\
\hline Simple surrogates $\ddagger$ & 0.56 & 0.55 \\
\hline
\end{tabular}

${ }^{\star}$ Number of events handling concentrates and uncorrected TWSS.

†Uses number of sheep dipped for concentrate and task by bath means for splash.

$\ddagger$ Uses average handling events for concentrate and task only means for splash. 
wore gloves. Questions were also asked about any regular use of OP compounds other than for dipping-such as pour on dips, and occupational and domestic insecticides.

Cumulative exposure indices

Cumulative exposure to OP sheep dips was derived from questionnaire responses. Variable OPEXP, was a cumulative version of the exposure model for a single dipping day in equation (2):

\section{$\mathrm{OPEXP}=\beta \times \mathrm{CONC}+\gamma \times \mathrm{SPLASH}$}

Parameters $\beta(=3.6)$ and $\gamma(=0.2)$ were chosen from the robust parameter set shown in table 3. Variables SPLASH and CONC were cumulative versions of exposure to dilute dip splash $(S)$ and concentrate $(C)$ in equation (2) using the simple surrogate model inputs. Variable SPLASH used task based mean splash scores:

$$
\begin{aligned}
S P L A S H=\sum_{j=1}^{f} N D_{j}\left(\frac{\% P L N G_{j}}{100} \times\right. \\
66+\frac{\% C H C K_{j}}{100} \times \\
\left.44+\frac{\% H E L P_{j}}{100} \times 10\right)
\end{aligned}
$$

where $\% \mathrm{PLNG}_{j}, \% C H C K_{j}$ and $\% H E L P_{j}$ were the estimated percentage of dipping in job $j$ spent working as a plunger, chucker, and helper respectively, and $N D_{j}$ is the number of dipping days spent in job $j$. This simple surrogate was easier to recall and had proved predictively no worse than the extended surrogate inputs that also included bath type. As circular baths were

DAYS

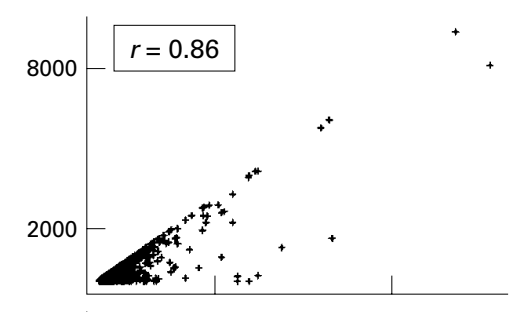

CONC
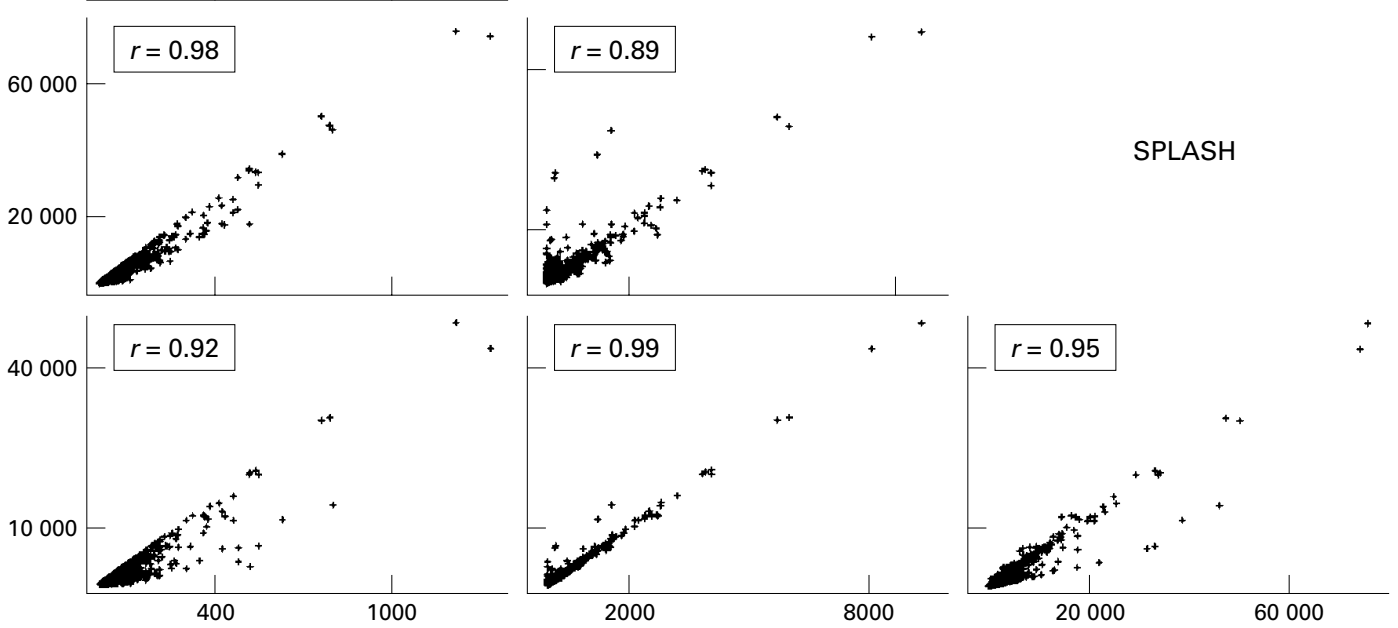

a relatively recent phenomenon and not common in the regions of the United Kingdom surveyed, and mobile baths relatively rare, task based splash scores assumed a linear bath.

Variable CONC, the cumulative number of events in which concentrate was handled, assumed a mean of eight handling events per day for principal handlers:

$C O N C=\sum_{j=1}^{f} N D_{j}\left(\frac{\% H A N D_{j}}{100} \times 8\right)$

where $\% H A N D j$ was the estimated number of occasions spent as principal concentrate handler in job $j$. Comparisons of surrogate input values had not warranted the use of flock size, which was also not easy for dippers to recall, to predict the number of handling events.

A simple cumulative exposure index, DAYS, the total number of days spent dipping sheep with OP dips since 1970, was also estimated:

$D A Y S=\sum_{j=1}^{f} N D_{j}$

Epidemiological study exposure estimates

In the epidemiological field study of sheep dippers, a cross section of 612 sheep dippers exposed to OP were interviewed. ${ }^{1}$ The distribution of total dipping days (DAYS) was highly skewed, with most subjects having fewer than 200 dipping days. The median was 54 days, with an interquartile range of between 28 and 102 days.

The four cumulative exposure indices described above were highly intercorrelated (fig 4), with a linear correlation of 0.92 between the

Figure 4 Scatter plot matrix of cumulative exposure indices among a cross section of sheep dippers showing linear correlation coefficients ( $r$ ). 
simple index DAYS and the index based on modelled exposure OPEXP. Correlation was least between DAYS and the cumulative events in which concentrate was handled, CONC $(r=0.86)$, reflecting the relatively large variation in exposure between dippers who were, and were not, principal concentrate handlers.

There was no significant linear correlation between age and days dipped $(r=0.06 ; p=0.15)$ or the other cumulative exposure indices. This was expected given the very small proportion of a working year devoted to dipping, the various changes in the legislation on the frequency of dipping since 1970, and the fact that many subjects were involved in dipping on a casual basis.

\section{Discussion}

A hygiene study of sheep dippers was used to derive an empirical exposure model based on the two principal sources of exposure, the handling of concentrate dip and direct or indirect splashing from dilute dip wash in the bath. In turn, dipping characteristics were sought which could explain differences in levels of exposure to the two sources and which were likely to be recalled retrospectively using an exposure history questionnaire. This two stage method produced a model that could be applied with a greater degree of confidence than a method based solely on a single stage regression modelling exercise that considered, simultaneously, all possible factors that might influence exposure. For example, the methodology described in this study was able to estimate the effects of concentrate handling and principal task on exposure, whereas a regression model that included both effects would suffer from collinearity due to their intercorrelation. The model derived in the this study was also robust to the fact that, although in this study plungers were most often the principal concentrate handlers, this was not always necessarily the case. ${ }^{8}$

The measure of actual exposure used in this study was a relatively crude measure based on the concentration of the metabolites DEP and DETP in the urine after dipping. It was an attempt to measure the total volume of diazinon absorbed by a dipper's body during the observed dipping session. Diazinon has the ability to penetrate intact skin and this was the principal route of exposure among dippers. Ideally a measure of the biologically relevant absorbed dose would be available but this would require sufficient biological monitoring data from individual workers to fit pharmacokinetic models which incorporated variation in uptake, metabolism and excretion rates between individual workers. The aim of this phase of the study, however, was to identify the important determinants of exposure that could also be used to estimate exposure retrospectively, based on recall, in the second, epidemiological, phase of the study. Despite the relatively small sample size, the method used was powerful enough to detect significant effects of exposure to concentrate, and simultaneously dilute dip splash, and allow estimation of parameters that weighted their relative contribution to overall exposure.
Concentrate handling was identified as the most important predictor of exposure and this confirmed an earlier observation that concentrate handlers had higher average concentrations of urinary metabolites after dipping than non-handlers. ${ }^{8}$ The relation was roughly linear suggesting that dermal exposure occurred consistently whenever concentrate was handled. Exposure to splashing of dilute dip wash in the bath was also found to be associated with exposure despite the difficulty in precisely measuring individual levels of dermal exposure. However, a method of splash scoring that was similar to the one used in earlier studies of sheep dippers ${ }^{812}$ again resulted in splash being correlated with measured exposure. Ingestion through the hands was not found to be a important route of exposure although estimation of exposure was difficult. Niven et $a l^{8}$ reported personal airborne exposures to diazinon among dippers at less than one tenth of the OES (occupational exposure standard, 8 hour time weighted average) of $0.1 \mathrm{mgm}^{-3}$, and therefore inhalation was not thought to be an important route of exposure.

In the epidemiological study, the questionnaire essentially identified six exposure scenarios for any recalled dipping session, corresponding to all combinations of the three principal tasks and an indicator of handling concentrate. Cumulative exposure was then estimated from the product of the exposure predicted by the model and the estimated number of dipping days within each of these scenarios, summed across all six exposure scenarios. For any person, predicted exposure would differ from true exposure by the extent to which the exposure predicted by the model differs from the person's historical mean exposure under each scenario, as well as recall error in the estimated number of dipping days. The effect of this measurement error in the cumulative exposure will have led to attenuation of the estimated parameters in exposureresponse regressions, ${ }^{1}$ reducing the power to detect a significant exposure gradient where one existed. ${ }^{13}$

Estimating the extent of the overall measurement error was difficult. The selection of a sample of dippers in the hygiene study, error in the measurement of true exposure during dipping among those selected, day to day and systematic variations in exposure of individual workers, and error in the recall of tasks and dipping days among those interviewed in the epidemiological study would all have contributed. Clearly, the exposure model was not able to predict unusually high exposures that have been found on occasions in this and other studies. However, reassurance of the validity of the method of predicting exposure can be drawn from the moderate, though significant, correlation between predicted and observed exposure among an independent sample of sheep dippers. This is particularly the case as variation in observed exposure between individual workers during a single session will tend to have been greater than variation in long term mean exposure between individual workers 
which is what the exposure model was only required to predict.

There is evidence that, among farmers, inaccurate recall of work related events-for example, exposure to pesticides and herbicides-is less of a problem than in workers generally. ${ }^{14}$ The dipping of a flock typically requires 1-3 days, and takes place once or twice a year on a seasonal basis. This infrequent but systematic pattern of dipping in particular lends itself well to retrospective estimation. Limited verification of recall was possible by comparing the temporal distribution of dipping days recorded in the epidemiological study to what was known about the historical use of OPs in dips. Of the total 55600 dipping days recorded, $35 \%$ were during the 8 years between 1984 and 1991 when OP dips were licensed and known to be widely used, and only $42 \%$ during the 13 years from 1970 to 1983 when OPs were less common in dip formulations. Also, only two factors, handling concentrates and dipping task, needed to be recalled for each dipping job. As one person was generally responsible for handling concentrate during dipping, this is likely to aid recall retrospectively. Recall of dipping task was also made easier by the fact that there were only three possible tasks and that task names were consistent across sheep farms visited.

No allowance was made in the model for use of protective clothing because no evidence of a significant reduction in exposure due to protective clothing was found. The power to detect such an effect was weak as very few subjects wore the recommended protective clothing throughout the dipping session. However, even in a controlled experiment to test the effectiveness of protective clothing, no evidence for a reduction in exposure was found with similar measurement methods, ${ }^{12}$ and it is possible for poor quality protective clothing to enhance dermal exposure by absorbing and retaining liquid close to the skin.

A method of estimating long term low level exposure to OP sheep dips with an exposure model has been described. This was principally an objective empirical method that did not rely on difficult subjective judgements on the effects of differing job characteristics on exposure. That the estimates of cumulative exposure based on this method among a cross section of dippers in the epidemiological field study were highly correlated with a simple estimate of total dipping days, or indeed any other cumulative exposure index, was to be expected and does not lessen the usefulness of the method. It did, however, suggest that the use of total dipping days to measure cumulative exposure was warranted, particularly as this variable would be prone to less measurement error and hence would be more powerful in exposure-response analyses. The lower correlation between total dipping days and cumulative exposure during handling concentrate was due to the importance of concentrate as a source of exposure among those who acted as concentrate handlers during dipping, relative to the many who dipped without exposure to concentrate and whose principal route of exposure was through the splashing of dilute dip from the dip bath.

We acknowledge the United Kingdom Health and Safety Executive, Department of Health, and Ministry of Agriculture, Fisheries and Food who jointly funded this study. Also, we thank all the farmers who took the time to participate in the study.

1 Pilkington A, Buchanan D, Jamal GA, et al. An epidemiological study of the relations between exposure to organophosphate pesticides and indices of chronic periphorganophosphate pesticides and indices of chronic peripheral neuropathy and neuropsychological abnormalities in sheep farmers and dippers. Occup Environ Med 2001;58:702-10.

2 Brouwer DH, Brouwer EJ, van Hemmen JJ. Estimation of long term exposure to pesticides. Am f Ind Med 1994;25:573-88.

3 Schneider T, Vermeulen R, Brouwer DH, et al. Conceptual model for assessment of dermal exposure. Occup Environ Med 1999;56:765-73.

4 London L, Myers JE. Use of a crop and job specific exposure matrix for retrospective assessment of long term exposure in studies of chronic neurotoxic effects agrichemicals. Occup Environ Med 1998;55:194-201.

5 Stephens R, Spurgeon A, Calvert IA, et al. Neuropsychological effects of long term exposure to organophosphates in sheep dip. Lancet 1995;345:1135-9.

6 Ray D. Organophosphorus esters: an evaluation of chronic neurotic effects. Leicester: MRC Institute for Environment and Health, 1998.

7 Rees H. Exposure to sheep dip and the incidence of acute symptoms in a group of Welsh sheep farmers. Occup Envisymptoms in a group of Wel
ron Med 1996;53:258-63.

ron Med 1996;53:258-63.
8 Niven KJM, Scott AJ, Hagen S, et al. Occupational hygiene assessment of sheep dipping practices and processes. Edinburgh: Institute of Occupational Medicine, 1993. (IOM report TM/93/03)

9 Nutley B, Cocker J. Biological monitoring of workers occupationally exposed to organophosphorous pesticides. Pesticide Science 1993;38:315-22.

10 Alessio L, Berlin A, Dell'Orto A, et al. Reliability of urinary creatinine as a parameter used to adjust values of urinary biological indicators. Int Arch Occup Environ Health 1985;55:99-106.

11 Genstat 5 Committee. Genstat 5 reference manual. Oxford: Clarendon Press, 1987.

12 Niven KJM, Hagen S, Scott AJ, et al. Occupational hygiene assessment of exposure to insecticides and the effectiveness of protective clothing during sheep dipping operations. Edinburgh: protective clothing during sheep dipping operations. Edinburgh: Institute of

13 Armstrong BK, White E, Saracci R. Principles of exposure measurement in epidemiology. Oxford: Oxford University Press, 1992.

14 Blair A, Zahm S. Methodological issues in exposure assessment for case-control studies of cancer and herbicides. $\mathrm{Am}$ F Ind Med 1990;18:285-93. 